\title{
Analysis of Energy Spectrum and Wave Function of Modified Poschl Teller Potential Using Hypergeometry and Supersymmetry Method
}

\author{
Fuzi Marati Solikhah ${ }^{1}$, Suparmi $^{2}$, and Viska Inda Variani ${ }^{3}$
}

\begin{abstract}
Modified Poschl Teller potential is a potential model used to describe behavior of inter moleculer forces. The spectrum energy and wave function of the particle affection in modified Poschl Teller potential are obtained by solving the Schrodinger equation, using hypergeometric equation and supersymmetric method. The energy spectrum and wave function are obtained from hypergeometric equation, which is constructed from Schrodinger equation through variable substitution. The energy spectrum and wave function are obtained using raising and lowering supersymmetric operator and applying shape invariance property. The solution of those equation of the energy spectrum and wave function obtained using these two methods are exact solution. Further, the wave function and density probability are visualized using computer simulation.
\end{abstract}

Keyword —energy spectrum, wave function, Modified Poschl Teller, hypergeometric, supersymmetric

Abstrak-Potensial Poschl Teller termodifikasi adalah model potensial yang digunakan untuk menerangkan fenomena gaya inter moleculer. Spektrum energi dan fungsi gelombang partikel yang dipengaruhi oleh potensial Poschl Teller termodifikasi dapat diperoleh dari penyelesaian persamaan Schrodinger, dengan menggunakan persamaan hypergeometry dan metode supersimetry. Spektrum energi dan fungsi gelombang diperoleh dari persamaan hypergeometry, yang mana persamaan tersebut didapat dari persamaan Schrodinger melalui subtitusi variabel. Spektrum energi dan fungsi gelombang juga bisa diperoleh dengan menggunakan raising dan lowering operator supersymmetry serta penerapan sifat-sifat shape invariance. Solusi dari persamaan spektrum energi dan fungsi gelombang yang diperoleh dengan menggunakan dua metode ini adalah penyelesaian eksak. Selanjutnya, fungsi gelombang dan rapat probabilitasnya divisualisasikan menggunakan simulasi komputer.

Kata kunci-spektrum energi, fungsi gelombang, Poschl Teller Termodifikasi, hipergeometri, supersimetri

\section{INTRODUCTION}

$\mathrm{S}$ chrodinger equation is the heart of quantum mechanics [1-2]. Energy spectrum and wave function of a particle system are obtained by solving the schrodinger equation [2] of the particle system directly. Schrodinger equation of potential systems can be solved exactly in which it has an important role in quantum mechanics. Energy spectrum and wave function are used to describe the behavior of subatomic particles. Energy spektum and wave function can be obtained from both the Schrodinger equation solution directly and indirectly. Direct solution is solved by reducing the differential equation of special functions [2-3] such as Hermit, Legendre, Besel, Laguerre and hypergeometry. However indirect solution can be solved using a supersimetry operators, equation WKB and SWKB approach. However, among of these functions are only differensial hypergeometry equation and supersimmetry approach that have the general form.

The hypergeometric equation can be used to solve exactly the Schrodinger equation for class of shape invariance potential and non shape invariance potential such as Wood-Saxon [4] and Hulthen potentials. The

Fuzi Marati Sholihah is with Post graduate program at Physics Department UNS, Jl. Ir. Sutami 36 A, Surakarta, Indonesia, , E-mail: fuzzimarati@yahoo.com.

Suparmi is with Department of Physics, FMIPA, UNS, Surakarta, Indonesia.

Viska Inda Variani is with Department of Physics, FMIPA, UNS, Surakarta, Indonesia. general form of second order differential equation of hypergeometric function is written as

$\left(z-z^{2}\right) \frac{d^{2} \phi}{d z^{2}}+(c-(a+b+1) z) \frac{d \phi}{d z}-a b \phi=0$

The general solution of the second-order differential equation of hypergeometric [3] expressed in the Equation 1 in example :

${ }_{2} F_{1}(a, b, c, z)=\sum_{n \sim 0} \frac{(a)_{n}(b)_{n}}{n !(c)_{n}}$

where,

$(a)_{n}=a(a+1)(a+2)(a+3) \ldots$

$(a)_{0}=1$

The energy spectrum system can be obtained by using a condition that is :

$a=-n$ or $b=-n$

It causes the hypergeometric series terminate so the finite solution of n-degree polynomial equation is obtained.

By using both Equations 1 and 2 it can be obtained the energy spectrum and wave function of modified Poschl Teller potential. The second order differential equation of hypergeometric function will be used to solve Schrodinger equation of modified Poschl Teller potential which is has effective potential is [4],

$V_{\text {eff }}=\frac{h^{2}}{2 m}\left\{\frac{\rho(\rho-1)}{\sinh ^{2} x}-\frac{\lambda(\lambda+1)}{\cosh ^{2} x}\right\}$

The schrodinger equation for a modified Poschl Teller potential is expressed as, 
$-\frac{h^{2}}{2 m} \frac{d^{2} \psi}{d x^{2}}+\frac{h^{2}}{2 m}\left\{\frac{\rho(\rho-1)}{\sinh ^{2} x}-\frac{\lambda(\lambda+1)}{\cosh ^{2} x}\right\} \psi=E \psi$

Equation 7 is reduced to the second order differential of hypergeometric function by variable substitution as follows [5].

$\cosh ^{2} x=z$

$\frac{\partial}{\partial x^{2}}=\frac{\partial}{\partial z} \frac{\partial x}{\partial z}=2 \sqrt{z(z-1)} \frac{\partial}{\partial x}$

$\frac{\partial^{2}}{\partial x^{2}}=2 \sqrt{z(z-1)} \frac{\partial}{\partial x} 2 \sqrt{z(z-1)} \frac{\partial}{\partial x}$

Further, by using Equation 8) we obtain,

$\frac{\partial^{2} \psi}{\partial x^{2}}=4 z(z-1) \frac{\partial^{2} \psi}{\partial z^{2}}+2(z-1) \frac{\partial \psi}{\partial z}$

We further the equations 8 and 9 into Equation 7 we obtained

$z^{2}-z \frac{\partial^{2} \psi}{\partial z^{2}}+\left(\frac{1}{2}-z\right) \frac{\partial \psi}{\partial z}+\left\{\frac{k^{2}}{4}-\frac{\rho(\rho-1)}{4(1-z)}-\frac{\lambda(\lambda+1)}{4 z}\right\} \psi=0$

Equation 10 can be changed into the second order differential hypergeometric function by using approximation solution around regular singular points at $\mathrm{z}=0$ and $\mathrm{z}=1$ of Equation 10. The approximate solution at $\mathrm{z}=0$ is

$\psi \sim z^{a}$

where, $\frac{-\lambda}{2}=\alpha$

The approximate solution at $\mathrm{z}=1$ is

$\psi \sim(1-z)^{\beta}$

It has been set that $\rho=2 \beta$

By combining these two solutions, we obtain the wave function parameter of modified Poschl Teller potential, namely:

$\psi=z^{\alpha}(1-z)^{\beta} f(z)$

$\frac{d \psi}{d z}=\alpha z^{\alpha-1}(1-z)^{\beta} f(z)-z^{\alpha} \beta(1-z)^{\beta-1} f(z)$

$+z^{\alpha}(1-z)^{\beta} f^{\prime}(z)$

$\frac{d^{2} \psi}{d z^{2}}=\alpha(\alpha-1) z^{\alpha-2}(1-z)^{\beta} f(z)-\alpha z^{\alpha-1} \beta(1-z)^{\beta-1} f(z)$

$+\alpha z^{\alpha-1}(1-z)^{\beta} f^{\prime}(z)-\alpha \beta z^{\alpha-1}(1-z)^{\beta-1} f(z)+\beta(\beta-1) z^{\alpha}$

$(1-z)^{\beta-1} f^{\prime}(z)+z^{\alpha}(1-z)^{\beta} f^{\prime \prime}$

$z(1-z) \frac{d^{2} f}{d z^{2}}+\left(\left(2 \alpha+\frac{1}{2}\right)-(2 \alpha+2 \beta+1) z\right) \frac{d f}{d z}$

$+\left(\frac{k^{2}}{4}-(\alpha+\beta)^{2}\right) f=0$

We further solution Equation 13 into Equation 10 we obtain the type hypergeometric Equation 16, By comparing Equation 16 and Equation 1 we obtain the energy spectrum and wave function, in example:

$E_{n}=-\frac{h}{2 m}(\lambda-\rho-2 n)^{2}$

$\psi=z^{\alpha}(1-z)_{2}^{\beta} F_{1}(a, b ; c, z)$
${ }_{2} F_{1}(a, b ; c, z)=\sum_{n=0} \frac{a_{n} b_{n}}{n ! c_{n}} z^{n}$

Supersymmetry (SUSY) is the symmetry retained under the transformation that interchange bosons and fermions. However it is a general belief that there are two distinet classes of particles which are not interchangeable, in example bosons and fermions. Supersymmetry however assumes that there are environments where bosons and fermions are indistinguishable. However, no evidences have been reported so far for the existence of the particles predicted by supersymmetric schemes [6] that means bosons could not interchange with fermions. Since bosons and fermions are clearly distinguishable in most of physical phenomena occurring around us. It is evident that even if SUSY is physically meaningful symmetry but it is definitely broken. According to Witten [6] SUSY quantum mechanics as a model of one dimensional field theory to understand how SUSY has been broken in nature. The key players in SUSY quantum mechanics Hamiltonian [7] is defined as SUSY quantum mechanics are supersymmetric charge operators in term of which the SUSY Hamiltonian may be expressed.

Although SUSY quantum mechanics it was introduced as a model for understanding SUSY breaking [8] it has started to walk alone independent of the original aim as a useful tool in non relativistic quantum mechanics. The application of SUSY to nonrelativistic quantum mechanics has result in better understanding of exactly solvable potential [8]. In particular, the notion of SUSY shape invariance provides a very elegant and cynthic way of presenting the operational method. The SUSY Hamiltonian is presented as

$H_{s s}=\left[\begin{array}{cc}-\frac{h^{2}}{2 m} \frac{d^{2}}{d x^{2}}+\frac{h^{2}}{\sqrt{2 m}} \frac{d \phi}{d x}+\phi & 0 \\ 0 & -\frac{h^{2}}{2 m} \frac{d^{2}}{d x^{2}}-\frac{h^{2}}{\sqrt{2 m}} \frac{d \phi}{d x}+\phi\end{array}\right]$

$=\left[\begin{array}{cc}H_{+} & 0 \\ 0 & H_{-}\end{array}\right]$

$H_{s s}$ is Hamiltonian supersymmetry.

$H_{ \pm}=\left[\begin{array}{cc}H_{+} & 0 \\ 0 & H_{-}\end{array}\right]$

$\left[\begin{array}{cc}H_{+} & 0 \\ 0 & H_{-}\end{array}\right]=\left[\begin{array}{cc}A A^{+} & 0 \\ 0 & A^{+} A\end{array}\right]$

Where $H_{+}$and $H_{-}$as the bosonic and fermionic supersymmtery partners in the Hamiltonian $\mathrm{H}_{ \pm}$. In the standard equation notation, the SUSY Hamiltonian can be written as,

$$
\begin{aligned}
& H_{-}=A^{+} A=-\frac{\hbar^{2}}{2 m} \frac{d^{2}}{d x^{2}}+V_{-}(x) \\
& H_{+}=A A^{+}=-\frac{h^{2}}{2 m} \frac{d^{2}}{d x^{2}}+V_{+}(x)
\end{aligned}
$$

where,

$V_{+}\left(x, a_{0}\right)=\phi^{2}+\frac{\hbar}{\sqrt{2 m}} \phi^{\prime}$ 


$$
\begin{aligned}
& V_{-}\left(x, a_{0}\right)=\phi^{2}-\frac{\hbar}{\sqrt{2 m}} \phi^{\prime} \\
& A^{+}=-\frac{\hbar}{\sqrt{2 m}} \frac{d}{d x}+\phi \\
& A=\frac{\hbar}{\sqrt{2 m}} \frac{d}{d x}+\phi
\end{aligned}
$$

$V_{+}$and $V_{-}$are the supersymmtery partners to each other, and $\phi$ is the superpotential. $\mathrm{A}^{+}$is a raising operator and A is a lowering operator.

The energy spectrum and wave function of one dimentional Hamiltonian can be derived algebraically using the notation of shape invariance and its relation to the factorization method. The SUSY Hamiltonian can be written as,

$H=\frac{-h^{2}}{2 m} \frac{d^{2}}{d x^{2}}+V(x)+\varepsilon$

It is always possible to perform a factorization of Hamiltonian as

$H=A^{+} A+\varepsilon$,

In which $\varepsilon$ is factorization energy or ground state energy. From Equation 21, 24, 27 and 28 we have:

$V_{\text {eff }}=V_{-}+\varepsilon$

$V_{e f f}=\phi^{2}-\frac{\hbar}{\sqrt{2 m}} \phi^{\prime}+\varepsilon$

$\phi$ is determined using Equation 29. By substituting Equation 21 into Equation 29 we set the superpotential of modified Poschl Teller

Superpotential is applied to determine the supersymmetric operator. $H_{+}$and $H_{-}$in 21 and 22 to determine the wave function. Equation 29 suggests that $\phi$ has the form as,

$\phi=D \tanh x+E \operatorname{coth} x$

where

$D=\frac{\hbar}{\sqrt{2 m}} \lambda$ and

$E=-\frac{\hbar}{\sqrt{2 m}} \rho$

Using Equations 26 and 30 we obtain

$\phi=\frac{\hbar}{\sqrt{2 m}} \lambda \tanh x-\frac{\hbar}{\sqrt{2 m}} \rho \operatorname{coth} x$

The first derivation of the superpotential is

$\phi^{\prime}=\left(\frac{h}{\sqrt{2 m}} \lambda \operatorname{sech}^{2} x+\frac{h}{\sqrt{2 m}} \rho\left(\operatorname{csch}^{2} x\right)\right)$

$\emptyset^{\prime}=\left(\frac{\hbar}{\sqrt{2 m}} \lambda \operatorname{sech}^{2} x+\frac{\hbar}{\sqrt{2 m}} \rho\left(\operatorname{csch}^{2} x\right)\right)$

The wave function we use:

$A \psi_{0}=0$

The exacted state wave function is obtained by using the property of raising operator i,e.i.,

$\psi_{n+1}\left(x, a_{0}\right)=A^{+} \psi_{n-1}\left(x, a_{1}\right)$

The energy spectrum is obtained by defining the notion of shape invariance potential in example:

$E_{n}^{-}=\sum_{k=1}^{n} R\left(a_{k}\right)$ where,

$R\left(a_{k}\right)=V_{+}\left(x, a_{k}\right)-V_{-}\left(x, a_{k+1}\right)$

To describe the behavior of particle then the wave function and probability density are visualized by computer simulation. The aim of the study energy spectrum and wave function using hypergeometry and supersymmetry method. The wave function and density probability are visualized using computer simulation.

We set up the values of $a_{0}=\rho, a_{0}=\lambda, a_{1}=\rho+1, a_{1}=\lambda-1$, Equation 18 and 19 is included on Equation 28, it is obtained the energy energy spectrum of modified Poschl Teller potential in Equation 37.

The wave function of modified Poschl Teller potential used to describe the graph. The aim of this study is firsty to determine the energy and the modified Poschl Teller potential. Secondy is to visualize graph of the wave function and the probability density using computer simulation. This study gives some advantages to the writer concerning with the wave function of modified Poschl Teller simulation and probability density. Simulation can facilitate in analyzing the probability of a particle in a certain position.

\section{METHOD}

Instrument used in this study is Software Delphi 7.0. The equations used are as follows [9-10].

\section{A. For Hypergeometric Equation Method.}

The wave function is obtained using Equation 13 and (14a)

$z(1-z) \frac{d^{2} f}{d z^{2}}+\left(\left(2 \alpha+\frac{1}{2}\right)=(2 \alpha+2 \beta+1) z\right) \frac{d f}{d z}$

$\left(\frac{k^{2}}{4}-(\alpha+\beta)^{2}\right) f=0$

$\psi=z^{\alpha}(1-z)_{2}^{\beta} F_{1}(a, b ; c, z)$

where, ${ }_{2} F_{1}(a, b ; c, z)=\sum_{n=0} \frac{a_{n} b_{n}}{n ! c_{n}} z^{n}$

$a=\alpha+\beta+\frac{k}{2}$

$b=\alpha+\beta-\frac{k}{2}$

$\alpha+\beta=-\frac{\lambda}{2}+\frac{\rho}{2}$

$c=2 \alpha+\frac{1}{2}$

$\psi=z^{\alpha}(1-z)^{\beta}{ }_{2} F_{l}(a, b ; c, z)$

1. To determine energy spectrum we use Equation 16 by setting

$\alpha+\beta+\frac{k}{2}=0$

B. For SUSY Operator Method

1. The wave function is obtained using Equations 25 , 26, 32, and 35 .

$A \psi_{0}=0$

$\psi_{n+1}\left(x, a_{0}\right)=A^{+} \psi_{n-1}\left(x, a_{1}\right)$

$A=\frac{h}{\sqrt{2 m}}\left(\frac{d}{d x}+\lambda \tanh x-\rho \operatorname{coth} x\right)$ 
$A^{+}=\frac{\hbar}{\sqrt{2 m}}\left(-\frac{d}{d x}+\lambda \tanh x-\rho \operatorname{coth} x\right)$

To determine energy spectrum we use Equation 36 and 37.

$E_{n}^{-}=\sum_{k=1}^{n} R\left(a_{k}\right)$

where,

$R\left(a_{k}\right)=V_{+}\left(x, a_{k}\right)-V_{-}\left(x, a_{k+1}\right)$

$V_{-}\left(x, a_{0}\right)=\frac{\hbar^{2}}{2 m}\left[\frac{\rho(\rho-1)}{\sinh ^{2} x}-\frac{\lambda(\lambda+1)}{\cosh ^{2} x}+(\lambda-\rho)^{2}\right]$

$V_{+}\left(x, a_{0}\right)=\frac{\hbar^{2}}{2 m}\left[\frac{\rho(\rho-1)}{\sinh ^{2} x}-\frac{\lambda(\lambda+1)}{\cosh ^{2} x}+(\lambda-\rho)^{2}\right]$

where,

$a_{0}=\rho, a_{0}=\lambda, a_{1}=\rho+1, a_{1}=\lambda-1$, then

$V_{-}\left(x, a_{1}\right)=\frac{\hbar}{2 m}\left[\frac{\rho(\rho-1)}{\sinh ^{2} x}-\frac{\lambda(\lambda+1)}{\cosh ^{2} x}+((\lambda-1)-(\rho+1))^{2}\right]$

Figure 1 shows that the flowchart of wave function and probability density. The wave function and probability density are visualized using computer simulation with $\lambda$ $=2$ and $\rho=-2$.

\section{RESUltS AND DisCUSSION}

The energy spectrum and wave function of modified Poschl Teller potential have been obtained by setting hypergeometric equation and supersymmetric operator when they are the same. Hypergeometric equation and supersymmetric method are usual algebraic method. So it is easier in their solution. The wave function is obtained using hypergeometric equation is derived from the Equation $13-15$ and 16 in example:

$$
\begin{aligned}
& \psi=(\cosh x)^{-\lambda}\left(-\sinh ^{2} x\right)_{2}^{2} F_{1}(a, b ; c, z) \\
& { }_{2} F_{1}(a, b ; c, z)=1+\frac{(-n)(-n)}{-\frac{3}{2}} \frac{z}{1 !}+\ldots
\end{aligned}
$$

The ground state wave function is obtained using Equation 39 for $n=0$ where ${ }_{2} F_{1}=1$. The state wave function of modified poschl Teller potential obtained using hypergeometric equation and SUSY operator is the same as depicted in Equation 41 and 42.

$$
\psi=(\cosh x)^{-\lambda}\left(-\sinh ^{2} x\right)^{\frac{\rho}{2}} 1+\frac{(-n)(-n)}{-\frac{3}{2}} \frac{z}{1 !}+\ldots
$$

$\psi_{0}=(\cosh x)^{-\lambda}\left(-\sinh ^{2} x\right)^{\frac{\rho}{2}}$

$\psi_{0}=c_{n}(\cosh x)^{-\lambda}-\sinh ^{\rho}$

For SUSY operator method, the wave function obtained by Equation 34 and 35. By using Equation 34 we get the grounds state of wave function.

$A \psi_{0}=0$

$\frac{\hbar}{\sqrt{2 m}}\left(\frac{d}{d x}+\lambda \tanh x-\rho \operatorname{coth} x\right) \psi_{0}=0$

$\frac{d \psi_{0}}{d x}=(-\lambda \tanh x-\rho \operatorname{coth} x) \psi_{0}$

The left and right side is integrated, it will be obtained.

$\int \frac{d \psi_{0}}{d x}=\int(-\lambda \tanh x-\rho \operatorname{coth} x) \psi_{0}$ $\ln \psi_{0}=-\lambda \ln \cosh x+\rho \ln \sinh x$

$\psi_{0}=c_{n}(\cosh x)^{-\lambda}(\sinh x)^{\rho}$

Figure 2 shows the graph of ground state wave function of modified Poschl Teller potential as function of angular potition $x, x$ in degree, as $\psi_{0}(x)$ in femto meter. Physically, this wave function has a meaning that at a ground state, it can be represented by a normalized wave function. The picture shows that the bigger angluler potition (x), the wave function produced will direct to unlimited. The graph form shows the cosines hyperbolic inverse function is multiplied by the sinus hyperbolic inverse function. For angular position more than \pm 150 degree wave function of Modified Poschl Teller potential is constant. The equation used in the simulation is $\psi_{0}=c_{n}(\cosh x)^{-\lambda}(\sinh x)^{\rho}$.

Figure 3 shows the graph of ground state wave function of modified Poschl Teller potential as function of angular potition $x, x$ in degree, as $\psi_{0}(x)$ in femto meter. Physically, this wave function has a meaning that at a ground state, it can be represented by a normalized wave function. The picture shows that the bigger angluler potition $(\mathrm{x})$, the wave function produced will direct to unlimited. The graph form shows the cosines hyperbolic inverse function is multiplied by the sinus hyperbolic inverse function. For angular position more than \pm 150 degree wave function of Modified Poschl Teller potential is constant. The equation used in the simulation is $\psi_{0}=c_{n}(\cosh x)^{-\lambda}(\sinh x)^{\rho}$. The wave function are visualized using computer simulation with $\lambda$ $=2$ and $\rho=-2$.

Figure 4 and 5 shows the graph of probability density of ground state wave functionas a function of angular potition $x,\left|\psi_{0}\right|^{2}$ in $\mathrm{fm}$ and $x$ in degree. Probability density is the representation of the square of absolute of the wave function. Probability density itself has a physical meaning as the opportunity of the existence of a particle in a certain area. The maximum value is on the top of the graph point. The cosinus hyperbolic inverse and sinus hyperbolic function is the main functions in the probability density graph. For the angular position more than \pm 150 degree probability density of Modified Poschl Teller potential is constant The equation used by simulation is in the Figure 4 and 5.

$$
\left|\psi_{0}\right|^{2}=\left|c_{n}(\cosh x)^{-\lambda}(\sinh x)^{\rho}\right|^{2}
$$

The modified Poschl Teller potential energy spectrum obtained by hypergeometric can be obtained by using a condition that is :

$$
\begin{aligned}
& a=-n \\
& a=a+\beta+\frac{k}{2} \\
& -\frac{\lambda}{2}+\frac{\rho}{2}+\frac{k}{2}=-n \\
& k=\lambda-\rho-2 n \\
& \frac{2 m}{h} E=-k^{2} \\
& E_{n}=-\frac{h^{2}}{2 m}(\lambda-\rho-2 n)^{2}
\end{aligned}
$$

The modified Poschl Teller potential energy spectrum obtained by supersymmetric operator method is: 


$$
\begin{aligned}
& V_{-}\left(x, a_{0}\right)=\frac{\hbar^{2}}{2 m}\left[\frac{\rho(\rho-1)}{\sinh ^{2} x}-\frac{\lambda(\lambda+1)}{\cosh ^{2} x}+(\lambda-\rho)^{2}\right] \\
& V_{+}\left(x, a_{0}\right)=\frac{h^{2}}{2 m}\left[\frac{\rho(\rho+1)}{\sinh ^{2} x}-\frac{\lambda(\lambda+1)}{\cosh ^{2} x}+(\lambda-\rho)^{2}\right]
\end{aligned}
$$

where, $a_{0}=\rho, a_{0}=\lambda, a_{1}=\rho+1, a_{1}=\lambda-1$

$V_{-}\left(x, a_{1}\right)=\frac{\hbar}{2 m}\left[\frac{\rho(\rho-1)}{\sinh ^{2} x}-\frac{\lambda(\lambda+1)}{\cosh ^{2} x}+((\lambda-1)-(\rho+1))^{2}\right]$

For SUSY operator method, the energy spectrum obtained by Equation 44, 45 and 46. By using Equation 36 and 37 we solved the energy spectrum of Modified Poschl Teller Potential.

$E_{n}=-\frac{h^{2}}{2 m}(\lambda-\rho-2 n)^{2}$

Table 1 shows that the energy spectrum of Modified Poschl Teller Potential and probability density. The energy spectrum are visualized using computer simulation. If the modified Poschl Teller potential energy at a ground state is zero, it can be analyzed that supersymmetry is in an undisturbed state

Table 2 Shows that the energy spectrum of Modified Poschl Teller Potential and probability density. The energy spectrum are visualized using computer simulation with $\lambda=2, \rho=-2$

\section{CONCLUSION}

The spectrum energy and wave function of modified Poschl Teller potential have been obtained using hypergeometric equation and supersymmetric operator method. The two methods provided the same result both in spectrum energy and at least ground state wave function. The supersymmetric operator method produce the spectrum energy and wave function easily without solving Schrodinger equation directly.

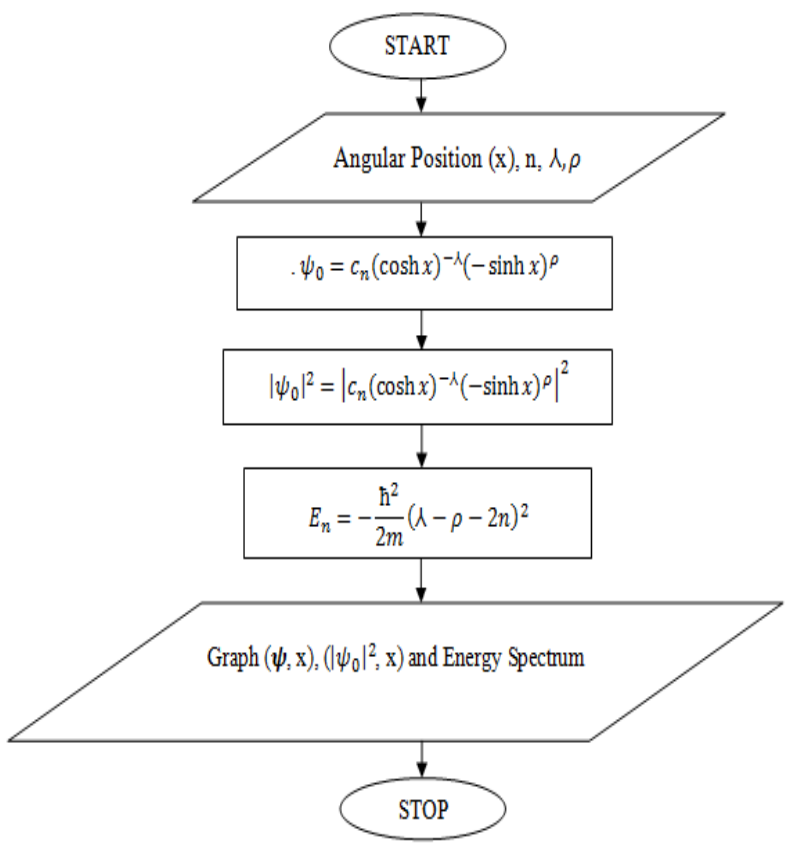

Figure 1. Flowchart of Modified Poschl Teller Potential Programming.

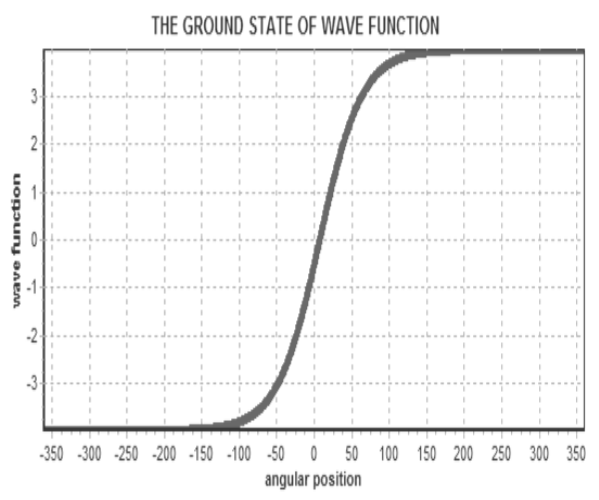

Figure 2. Ground state wave function modified Poschl Teller Potential as a function of angular position $\mathrm{x}$ in degree and (wave function) $\psi_{0}$ in fm with $\lambda=2$ and $\rho=2$

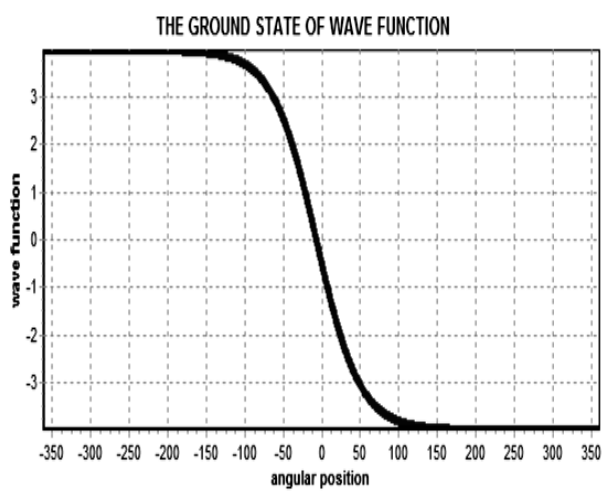

Figure 3. Ground State Wave Function Modified Poschl Teller Potential as a function of angular position $\mathrm{x}$ in degree and (wave function) $\psi_{0}$ in fm with $\lambda=2$ and $\rho=2$ 


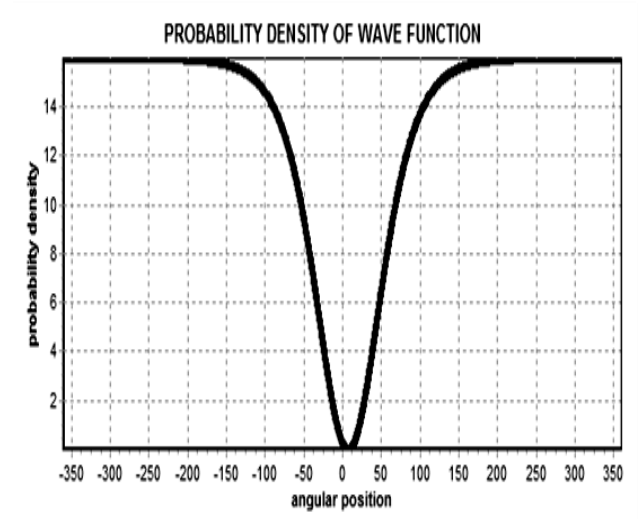

Figure 4. Probability density of ground state of wave function as a function of angular position $\mathrm{x}$ in degree and (probability density) $\left|\psi_{0}\right|^{2}$ in fm with $\lambda=2$ and $\rho=2$

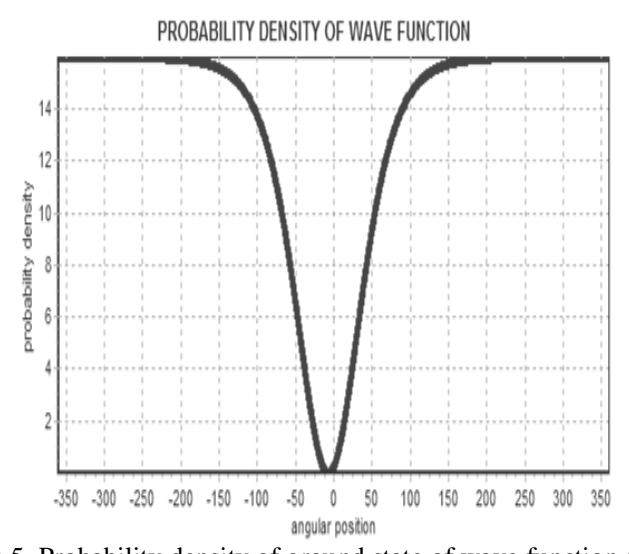

Figure 5. Probability density of ground state of wave function as a function of angular position $\mathrm{x}$ in degree and (probability density $\left|\psi_{0}\right|^{2}$ in fm with $\lambda=2$ and $\rho=2$

TABLE 1.

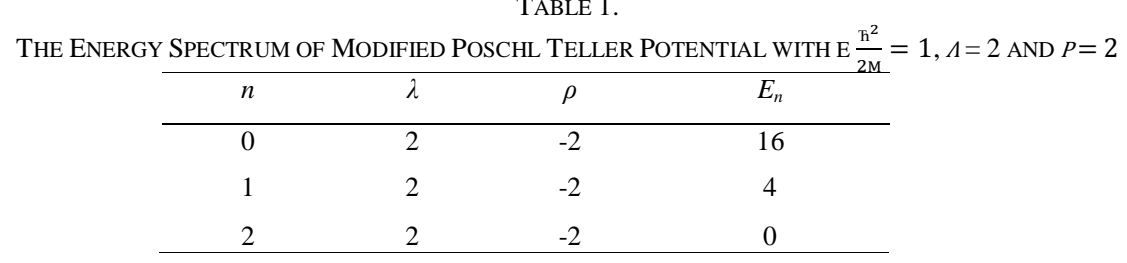

TABLE 2.

\begin{tabular}{|c|c|c|c|}
\hline$n$ & $\lambda$ & $\rho$ & $E_{n}$ \\
\hline 0 & 2 & 2 & 0 \\
\hline 1 & 2 & 2 & -4 \\
\hline 2 & 2 & 2 & -16 \\
\hline 3 & 2 & 2 & -36 \\
\hline 0 & -2 & 2 & 0 \\
\hline 1 & -2 & 2 & --4 \\
\hline 2 & -2 & 2 & -16 \\
\hline 3 & -2 & 2 & -36 \\
\hline
\end{tabular}

\section{REFERENCES}

[1] D. Donald, Principles Of Quantum Mechanics, Cambridge, University Press, 2002.

[2] S. Trachanas, Quantum Mechanical Potentials Exactly Solvable In Term Of Higher Hypergeometric Function I: The third-order Case, (FROTH) and Department Of Physics, University of Crete, Crete, Greece, 2011.

[3] A. N. Ikot, Awoga, L. E. Akpabio, and B. Ita, Solution of Schrodinger Equation with Generalized inverted Hyperbolic Potensial, Department of Physics, University of Uyo, Nigeria, 2005.

[4] J. S. Dehesa and Sokorin, Information-theoretic measure for Morse and Poschl-Teller potentials, Department of Physics, Granada University, Spain, (1-3), 2005.
[5] A. N. Ikot and Akpabio, Applied Physics Research, Approximate Solution of the Schrodinger Equation with Rosen Morse Potensial Including the Centrifugal, Term, Vol 2, No 2, Department of Physics, University of Uyo, Nigeria, 2010.

[6] M. Dine, Supersymmetry And String Theory Beyond the Standart Model, Cambridge University Press, Ukraina, 2007.

[7] C. Benjamin, C. Jasso, and M. kirbhbach, The Trigonometric Rosen Morse Potential as a Prime Candidate For an Effectif QDC Potential, Autonomous University, Mexico, 2005.

[8] S. Meyur, Electronic Journal of Theoritical Physics, Algebraic Aspects for Two Solvable Potentials, EJPT 25 271-224, Bengal, India, 2011.

[9] Wahana Komputer, Pemrograman Borland Delphi 7.0, Andi Offset, Yogyakarta, 2003.

[10] H. P. William, T. A. Saul, and V.T. William, Numerical Receipes the Art of Scientific Computing, Cambridge University press, 2007. 